\title{
ALDRICH KIDWELL (Peggy), ACKERBERG-HASTINGS (Amy), LINDSAY ROBERTS (David), Tools of American Mathematics Teaching, 1800-2000
}

Baltimore : Johns Hopkins University Press, 2008, 418 p. (coll. « Johns Hopkins Studies in the History of Mathematics »)

\section{Caroline Ehrhardt}

\section{OpenEdition}

Journals

\section{Édition électronique}

URL : https://journals.openedition.org/histoire-education/2308

DOI : 10.4000/histoire-education.2308

ISSN : 2102-5452

\section{Éditeur}

ENS Éditions

\section{Édition imprimée}

Date de publication : 1 janvier 2011

Pagination : 88-91

ISSN : 0221-6280

Référence électronique

Caroline Ehrhardt, «ALDRICH KIDWELL (Peggy), ACKERBERG-HASTINGS (Amy), LINDSAY ROBERTS (David), Tools of American Mathematics Teaching, 1800-2000», Histoire de l'éducation [En ligne], 129 | 2011, mis en ligne le 09 janvier 2012, consulté le 20 mai 2021. URL : http://journals.openedition.org/ histoire-education/2308 ; DOI : https://doi.org/10.4000/histoire-education.2308

Ce document a été généré automatiquement le 20 mai 2021.

(c) Tous droits réservés 


\section{ALDRICH KIDWELL (Peggy), ACKERBERG-HASTINGS (Amy), LINDSAY ROBERTS (David), Tools of American Mathematics Teaching, $1800-2000$}

Baltimore : Johns Hopkins University Press, 2008, 418 p. (coll. « Johns Hopkins Studies in the History of Mathematics »)

\section{Caroline Ehrhardt}

\section{RÉFÉRENCE}

ALDRICH KIDWELL (Peggy), ACKERBERG-HASTINGS (Amy), LINDSAY ROBERTS (David), Tools of American Mathematics Teaching, 1800-2000, Baltimore : Johns Hopkins University Press, 2008, 418 p. (coll. « Johns Hopkins Studies in the History of Mathematics »)

1 Tools of American Mathematics Teaching constitue une étude historique des objets qui ont formé, au cours des deux cents dernières années, la « culture matérielle » des cours de mathématiques aux États-Unis, de la maternelle à l'université. L'ouvrage est divisé en 18 chapitres en grande partie indépendants, organisés en quatre parties. Dans la première partie, les auteurs examinent ce qu'ils nomment les « outils de présentation et de pédagogie générale ». Ce sont des objets que l'on pouvait utiliser pour enseigner les mathématiques, mais qui ne leur étaient pas spécifiques, comme les manuels (chap. 1), le tableau noir (chap. 2), les tests standardisés (chap.3), le rétro-projecteur (chap. 4) et les "machines à apprendre » (teaching machines, chap. 5). Les trois parties suivantes présentent des objets exclusivement destinés à l'enseignement des mathématiques. Ainsi, la seconde partie est consacrée aux instruments de calcul: abaque (chap.6), règle à calculs (chap.7), cube pour extraire des racines cubiques 
(chap. 8) et solides en bois pour apprendre à compter (chap. 9). Dans la troisième partie, les auteurs s'intéressent aux instruments de mesure et de représentation : sont successivement étudiés le rapporteur (chap.10), un ensemble d'objets destinés à familiariser les élèves avec le système métrique (chap.11), le papier millimétré (chap.12), les modèles de solides géométriques (chap.13) et les règles articulées (chap. 14). La quatrième et dernière partie, enfin, étudie les technologies informatiques, comme les machines à calculer (chap. 15), les ordinateurs (chap. 16 et 17) et les logiciels et calculatrices graphiques (chap. 18).

2 Dans chacun des chapitres, les auteurs donnent une description détaillée de l'objet luimême et de ses évolutions, illustrée par de nombreuses photographies. La richesse des archives mobilisées va toutefois bien au-delà de la collection mathématique du National Museum of American History (Washington DC) dont la plupart de ces images sont issues. En effet, le livre s'appuie également sur de très nombreuses sources primaires et secondaires, qui permettent d'inscrire l'histoire matérielle de ces objets dans l'histoire de l'éducation comme dans celle des mathématiques. Les instruments mathématiques permettent ainsi, à partir d'études de cas précises et circonstanciées, d'aborder les changements du système scolaire américain (chap. 1, 3 et 10), les conséquences des bouleversements économiques, politiques et technologiques sur l'éducation (chap. 15 à 18), l'évolution des mathématiques (chap. 7 et 8) et celle des idées sur l'enseignement (chap. 3), ou encore les effets de la circulation des personnes sur celle des savoirs et des pratiques (chap. 2, 6 et 7). Le chapitre 9, consacré aux dispositifs matériels que l'on donne à manipuler aux enfants pour qu'ils apprennent à compter, fournit ainsi un exemple particulièrement abouti des interactions entre changements sociaux, politiques gouvernementales, théories pédagogiques et fabricants industriels dans l'adoption d'un nouvel objet dans les salles de classes.

3 L'un des grands mérites du livre est de parvenir à prendre en compte ces contextes sociaux, culturels et technologiques tout en maintenant l'analyse au niveau des acteurs eux-mêmes. Dans de nombreux chapitres, en effet, les auteurs mettent en avant le rôle fondamental joué par les enseignants soucieux d'innovations pédagogiques et les inventeurs, tel R. P. Baker qui, après avoir lu un article de recherche sur les coniques en 1903, a fabriqué une série de modèles géométriques qu'il a passé sa vie à promouvoir (chap.13). On peut toutefois regretter que cette attention accordée aux acteurs ne s'étende pas aux bénéficiaires de toutes ces innovations pédagogiques : sauf dans les chapitres de la première partie, qui portent sur des objets utilisés dans des cours de mathématiques sans être conçus spécifiquement à cet effet, on n'apprend finalement que peu de choses sur la façon dont les instruments ont pu intervenir effectivement dans l'apprentissage des élèves.

4 Étant donné la somme considérable de documents et d'informations rassemblés et la qualité de l'ouvrage, qui feront très certainement de lui un livre incontournable en son domaine, on aurait aimé que les auteurs présentent des conclusions plus générales sur le rôle joué, collectivement, par les instruments mathématiques dans l'enseignement de cette discipline. Cela aurait permis, en particulier, de revenir sur certains thèmes récurrents que l'on retrouve ça et là au cours des chapitres, et qui ne constituent pas le moindre intérêt du livre. Il s'agit, d'une part, de la tension entre l'abstraction des contenus et la manipulation d'objets matériels, qui fait écho aux débats qui traversent toute la période contemporaine entre méthodes empiriques et synthétiques dans les mathématiques et dans leur enseignement, et, d'autre part, des interactions entre deux 
échelles d'analyse, celle de l'État et celle de la salle de classe : celles-ci transparaissent, par exemple, dans l'étude de la pérennisation ou de l'abandon de certains instruments, ou dans l'évocation des adaptations imposées, au sein même de chaque salle de classe, par l'évolution générale du système éducatif.

5 Malgré ces quelques réserves, Tools of American Mathematics Teaching s'imposera certainement, grâce à la richesse de ses sources et à la variété des instruments qu'il présente, comme un ouvrage de référence en dehors des États-Unis pour ceux qui chercheront des informations sur l'un des dix-huit objets auxquels il est consacré, que ce soit dans le cadre de la recherche en histoire des mathématiques ou en histoire de l'éducation. 